\title{
The Frank-Starling Law: a jigsaw of titin proportions
}

\author{
Vasco Sequeira $^{1}$. Jolanda van der Velden ${ }^{1,2}$
}

Received: 5 April 2017 / Accepted: 23 May 2017 / Published online: 21 June 2017

(C) The Author(s) 2017. This article is an open access publication

\begin{abstract}
The Frank-Starling Law dictates that the heart is able to match ejection to the dynamic changes occurring during cardiac filling, hence efficiently regulating isovolumetric contraction and shortening. In the last four decades, efforts have been made to identify a common fundamental basis for the Frank-Starling heart that can explain the direct relationship between muscle lengthening and its increased sensitization to $\mathrm{Ca}^{2+}$. The term 'myofilament length-dependent activation' describes the length-dependent properties of the myofilaments, but what is(are) the underlying molecular mechanism(s) is a matter of ongoing debate. Length-dependent activation increases formation of thick-filament strongly-bound cross-bridges on actin and imposes structural-mechanical alterations on the thin-filament with greater than normal bound $\mathrm{Ca}^{2+}$. Stretch-induced effects, rather than changes in filament spacing, appear to be primarily involved in the regulation of length-dependent activation. Here, evidence is provided to support the notion that stretch-mediated effects induced by titin govern alterations of thick-filament force-producing cross-bridges and thin-filament $\mathrm{Ca}^{2+}$-cooperative responses.
\end{abstract}

Keywords Frank-Starling · Titin · Cross-bridges ·

$\mathrm{Ca}^{2+}$-cooperativity

This article is part of a Special Issue on 'Titin and its Binding Proteins in Striated Muscles' edited by Amy Li and Cristobal G. dos Remedios.

Vasco Sequeira

v.sequeiraoliveira@vumc.nl

1 Department of Physiology, Institute for Cardiovascular Research, VU University Medical Center, $\mathrm{O} \mid 2$ building, $11 \mathrm{~W}-53$, De Boelelaan 1118, 1081 HV Amsterdam, the Netherlands

2 ICIN- Netherlands Heart Institute, Utrecht, The Netherlands

\section{The Frank-Starling Law}

Building on the work of their German and British predecessors, Otto Frank and Ernest Starling at the turn of the twentieth century described the relationship between the length of the heart fibers and its power of contraction, the so-called FrankStarling Law of the Heart (Sequeira and van der Velden 2015). This relationship enables beat-to-beat adjustment of cardiac output in response to changes in venous pressure. At the single cardiac cell level, the Law mandates that there is a direct relationship between myofilament length and their sensitivity to $\mathrm{Ca}^{2+}$ ions, such that more force is generated at a given concentration of $\mathrm{Ca}^{2+}$ as fibers are lengthened. The term 'myofilament length-dependent activation' describes the length-dependent properties of the myofilaments. It has been attributed to structural-mechanical alterations of the thin-filament with greater than normal bound $\mathrm{Ca}^{2+}$ and to the increased formation of strongly-bound cross-bridges on actin, following muscle stretching (Hibberd and Jewell 1982; Allen and Kentish 1985; Sequeira and van der Velden 2015). Moreover, myofilament length-dependent activation has been shown to be additionally, and importantly, regulated by the degree of contractile protein phosphorylation (Konhilas et al. 2003; Hanft et al. 2013; Sequeira et al. 2013; Wijnker et al. 2014).

\section{The role of interfilament spacing}

Research on interfilament lattice spacing suggested it played a primary role in myofilament length-dependent activation. This concept dominated the literature for two decades until the late 1990s. Evidence emerged that supported the notion that myocyte lengthening was accompanied by an increased sensitization of the thin-filaments to $\mathrm{Ca}^{2+}$, and that a reduction in the distance between the myosin cross-bridges and actin 
filaments facilitated actomyosin interaction. In 1977, Godt and Maughan (1977) used the high-molecular-weight polymer (dextran) to reduce the interfilament distance, thereby varying maximum tension levels of $\mathrm{Ca}^{2+}$-activated skeletal muscle fibers. Because dextran was unable to diffuse between the myofilaments, it osmotically compressed them, thereby decreasing the distance of their lattice.

These results were confirmed in consecutive studies, and by other groups, using either skeletal (Godt and Maughan 1981; Moss et al. 1983; Martyn and Gordon 1988; Wang and Fuchs 1995) or cardiac (McDonald and Moss 1995; Wang and Fuchs 1995) muscle preparations, adding support to the idea that lattice spacing, rather than changes in length, was responsible for the changes in myofilament $\mathrm{Ca}^{2+}$-sensitivity. These findings were in good agreement with the proposition of Hofmann and Fuchs (1987a, b) and Fuchs and Wang (1996) who showed that both $\mathrm{Ca}^{2+}$-sensitivity and $\mathrm{Ca}^{2+}$-affinity to cardiac troponin $\mathrm{C}$ $(\mathrm{cTnC})$ were directly correlated with changes of lattice spacing but not with sarcomere length in cardiac muscle.

\section{Is $\mathrm{Ca}^{2+}$-sensitivity directly related to changes in myofilament lattice?}

Despite the large amount of evidence supporting the lattice spacing mediated-effects as the primary mechanism involved in length-dependent activation, filament lattice alterations were never directly measured when dextran was added to the muscle preparations. Instead, indirect assessment was based on the assumption that lattice alterations nearly correlated with changes of muscle width. Direct visualization of changes to interfilament lattice spacing as a function of dextran application, was published in 2000-2003 by the Pieter de Tombe laboratory (Irving et al. 2000; Konhilas et al. 2002b, 2003), who used synchrotron X-ray diffraction in membranepermeabilized and intact cardiac tissue. They showed that myofilament $\mathrm{Ca}^{2+}$-sensitivity was not linearly related to changes in interfilament spacing as a result of osmotic compression by dextran. Instead, compression of the lattice, observed when the sarcomere length was increased to optimal length $(2.2 \mu \mathrm{m})$, did not affect myofilament $\mathrm{Ca}^{2+}$-sensitivity (Konhilas et al. 2002b, 2003). Konhilas et al. (2002b, 2003) suggested that lattice changes are at least secondary in lengthdependent activation.

\section{How does stretch activate contraction?}

If not by lattice spacing alterations, how does stretching or increasing fiber length govern length-dependent activation and the Frank-Starling effect? What is at the heart of the Frank-Starling "jigsaw"? Overall, not one, but several, synergistically mechanisms, mediated by the giant protein titin following fiber stretch, appear to drive enhanced $\mathrm{Ca}^{2+}$ bound to cardiac troponin $\mathrm{C}$ (cTnC). The formation of increased numbers and/or more strongly-bound cross-bridges on actin increase the cooperation of force-producing thin-filament units. Stretch-induced effects, rather than changes in filament spacing, mediated by titin, appear to be primarily involved in the regulation of length-dependent activation. In this review, evidence is presented that supports the idea that upon stretchbecause it is anchored to the Z-disk-titin can not only act on thick-filament structures (and therefore is in a good position to orchestrate direct changes on cross-bridges) but also on thinfilament functional units (actin-tropomyosin-troponin).

\section{Titin-mediated recruitment of thick-filament cross-bridges}

Evidence from the last decade revealed that the giant elastic protein titin may serve as a length-dependent mechanosensor. Titin is a huge (3.8 MDa) protein that extends from the Z-disc to the M-band (Maruyama et al. 1985; Fürst et al. 1988). Its Nterminal region interacts with actin in the Z-disc (Trombitás et al. 1997). The I-band region of titin is extensible and consists of three elastic components that act as a spring (Labeit and Kolmerer 1995). In the A-band, titin is relatively inextensible because it interacts with the thick-filament proteins, such as the light meromyosin portion of myosin and cardiac myosin-binding protein $\mathrm{C}$ (cMyBP-C). Titin then links up with titin in the opposite half-sarcomere at the M-line (Maruyama et al. 1985; Freiburg and Gautel 1996; Zoghbi et al. 2008). Titin may exert its effects in two ways. Its primarily effect seems to involve thick-filament cross-bridge recruitment, and its secondary effect is to decrease the filament lattice to approximate myosin and actin filaments.

Stretch appears to exert its titin-dependent length activation by potentiating the recruitment of rested-to-ready stronglybound cross-bridges. X-ray structural studies has provided evidence that diastolic lengthening rearranges thick-filament structures, such that more cross-bridges are recruited for activation (Ait-Mou et al. 2016). Increased cross-bridge recruitment is directly associated with myofilament lengthdependent activation (Ait-Mou et al. 2016). Because titin is obliquely oriented to the sarcomere axis and attaches both myosin and cMyBP-C (Maruyama et al. 1985; Freiburg and Gautel 1996; Zoghbi et al. 2008), it will impose a passive strain on thick-filament proteins, thereby changing the geometry of cross-bridges (Fukuda et al. 2000, 2001; Ait-Mou et al. 2016). In frog skeletal muscle, sarcomere lengthening increases myosin periodicity (Wakabayashi et al. 1994), such that the transition of the population of myosin cross-bridges from rested ("Off") to weakly-bound increases (i.e., from an orderly to a disorderly state with respect to the axis of the thick-filament) (Fig. 1) (Malinchik et al. 1997; Xu et al. 


\section{a}
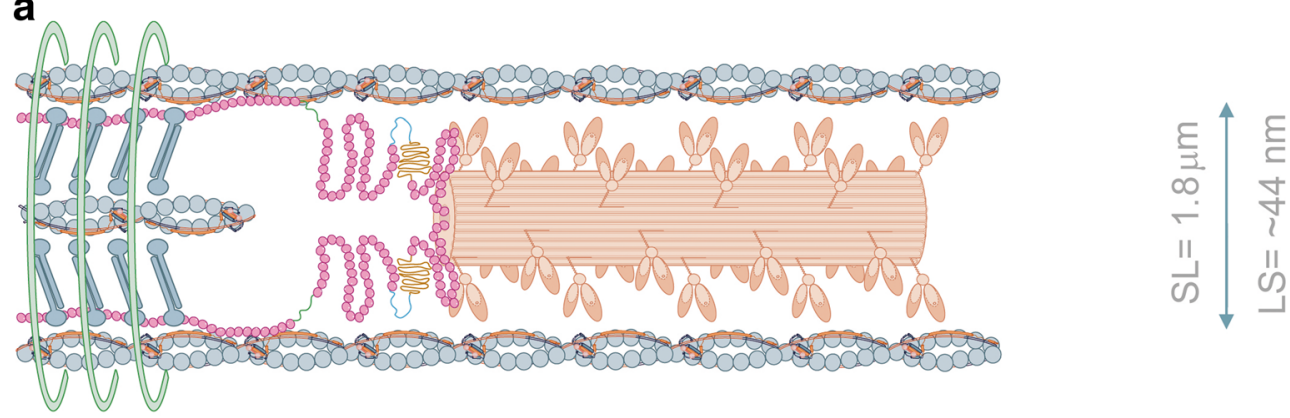

\section{b}

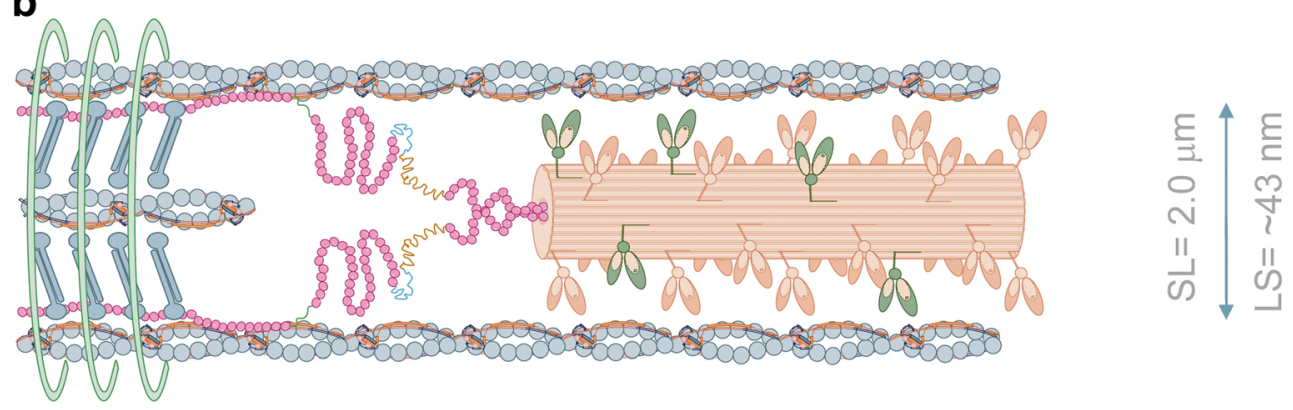

\section{C}

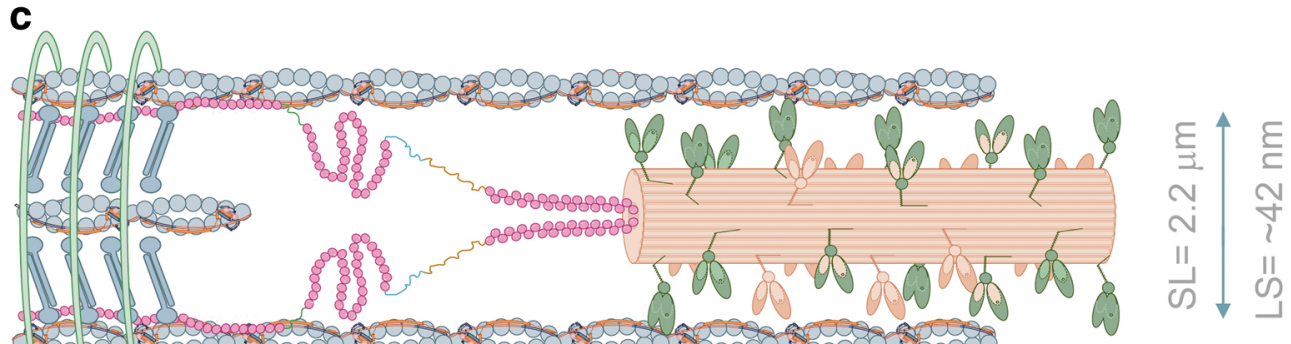

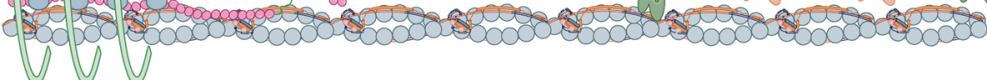
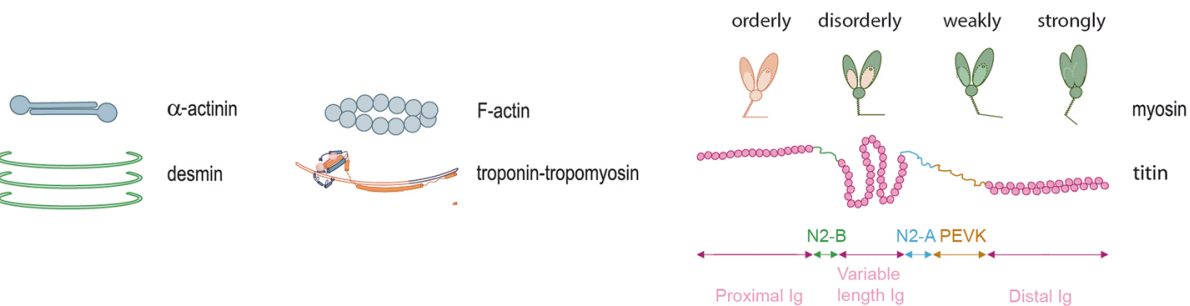

Fig. 1 A schematic model of half-sarcomere at varying sarcomere lengths at low $\mathrm{Ca}^{2+}$ conditions. Lattice spacing dimensions at each varying length were taken from Konhilas et al. (2002a). As the muscle is stretched from a relatively short sarcomere length (a) to higher sarcomere lengths $(\mathbf{b}, \mathbf{c})$, lattice spacing becomes smaller, myosin approximates to actin and cross-bridges transit from orderly into disorderly states. The I-band region of titin is the extensible region and consists of three elastic components that act as a spring element: (1) tandem immunoglobulin (Ig)-like domain regions, with proximal (near Z-disc) and distal (near I-A regions) segments; (2) the PEVK sequence-region rich in proline $(\mathrm{P})$, glutamic acid $(\mathrm{E})$, valine $(\mathrm{V})$ and lysine $(\mathrm{K})$; and (3) the N2B and N2BA elements (both isoforms contain N2B segments, but only the N2BA isoform contains an additional N2A

1997). A similar finding is observed in cardiac muscle where the orientation of myosin heads becomes more perpendicular element) (Labeit and Kolmerer 1995). Titin-induced stretch imposes a passive strain on the thick-filament proteins, which reduces lattice spacing and changes the arrangement of cross-bridges. Upon fiber lengthening, titin anchorage both on actin and tropomyosin at the I-band region, imposes a passive strain on thin-filament proteins and increases the cooperative unit size. Please note that more myosin motors are turned "On" during systolic activation (Reconditi et al. 2017). Distinct myosin colors are depicted to better illustrate the transition of orderly to disorderly projections. $\alpha$-actinin and desmin illustrate the Z-disc border. Note: cardiac myosin-binding protein $\mathrm{C}$ (cMyBP-C) was omitted to simplify the drawing and the width and sarcomere length dimensions are not to scale. (image adapted from Sequeira and van der Velden 2015)

to the thick-filament axis when sarcomere length is increased (i.e., the proportion of cross-bridge motors switched "On" 
increases upon stretch) (Farman et al. 2011). These studies suggest that stretch-induced activation by titin imposes a radial strain over the thick-filament, which recruits rested-to-ready force-generating cross-bridges under low $\mathrm{Ca}^{2+}$-activation conditions (Fig. 1). The magnitude of this effect is dependent on the rise of systolic stress, such that, at high filament loads during systolic activation, this mechanosensing ability recruits more force-producing cross-bridges (Linari et al. 2015; Reconditi et al. 2017). In other words, the fraction of "super relaxed" cross-bridge units transiting from the Off to the On state increases with thick-filament stress (Kampourakis et al. 2016; Reconditi et al. 2017).

Although a reduced filament lattice spacing may not be primarily involved in length-dependent activation, one cannot entirely exclude the prospect that lattice spacing reductions may secondarily exacerbate the effects of fiber lengthening (Fig. 1). An increase in titin-based passive tension reduces filament lattice upon stretch and approximates myosin and actin. A correlation between enhanced length-dependent activation and higher levels of passive tension has been reported (Cazorla et al. 1999, 2001; Fukuda et al. 2003). The concept is also supported by a recent report using a rat model expressing a giant titin isoform, which clearly associates reduced myofilament force development and impaired length-dependent activation (Mateja et al. 2012). Hence, reductions in the titininduced changes in lattice bring the cross-bridges closer to the thin-filaments, thus favoring force development of $\mathrm{Ca}^{2+}$-activated fibers.

\section{Titin-induced strain on the thick-filament increases Off-On transition of thin-filament units}

Length-dependent activation has been associated with titin-induced strain on the thick-filament and the subsequent potentiation of Off-On transition of thin-filament units (the steric blocking-unblocking model) (Terui et al. 2008; Farman et al. 2010; Mateja et al. 2012). There is evidence to suggest that length-dependent activation is regulated via an Off-On switch of the thin-filament, potentiated by the greater movement of tropomyosin on actin that uncovers more myosin-binding sites (Smith and Fuchs 1999). In the absence of $\mathrm{Ca}^{2+}$ (B-state), tropomyosin sterically blocks the myosin-binding sites on actin. In cardiomyocytes, a rise in $\mathrm{Ca}^{2+}$ induces a conformational change in the troponin-tropomyosin complex, resulting in the movement of tropomyosin on actin which exposes most of its myosin-binding sites (C-state). Weakly-bound cross-bridges populate the $\mathrm{C}$-state (myosin-ADP-Pi). Transition to the myosin-induced state and muscle activation (M-state) involves the release of Pi from crossbridges and the formation of strong-binding cross-bridges (myosin-ADP). This induces additional movement of tropomyosin, resulting in contraction and myofilament sliding. The relevance of the transition from the B- to the $\mathrm{C}$-state for proper length-dependent activation was shown more than two decades ago by Smith and Fuchs (1999), who were the first to provide evidence for a lengthsensitive step in the Off-On transition of thin-filament units. A reduction in ionic strength $(<0.05 \mathrm{M})$ was known to shift the B- to the $\mathrm{C}$-state equilibrium (where the population of recruited cross-bridges increases; $\mathrm{Xu}$ et al. 1987; Head et al. 1995) coincided with impaired lengthdependent activation (Smith and Fuchs 1999). Terui et al. (2008) subsequently demonstrated that length-dependent activation is associated with titin-induced strain on the thick-filament and the troponin complex. They reconstituted cardiac thin-filaments with fast skeletal troponin and observed reduced length-dependent activation to levels similar to that of skeletal muscle. In turn, reconstitution with $\mathrm{cTn}$ restored length-dependent activation, which they associated with increased transition from the B- to the C-state (Terui et al. 2008).

\section{Similarities to insect flight muscle}

Recent findings from stretch-activation of insect flight muscle support the view that Off-On thin-filament transitions are central to the length-dependent response. Activation of these muscles requires a stretch-activation mechanism in addition to $\mathrm{Ca}^{2+}$. Because stretching increases the periodicity of myosin (and a re-orientation of myosin heads on the thick-filament; Wakabayashi et al. 1994; Farman et al. 2011), insect flight muscle activation also seems to require the formation of strongly-bound cross-bridges recruited by stretch and $\mathrm{Ca}^{2+}$-binding to $\mathrm{TnC}$, in order to potentiate full movement of tropomyosin and accessibility of myosinbinding sites. In support, Perz-Edwards et al. (2011) recently confirmed this proposition by demonstrating that stretchactivation of insect flight muscle requires the steric blocking-unblocking model (Off-On thin-filament transition), such that strong-binding cross-bridges are secondarily recruited to induce the movement of tropomyosin. This study is also interesting because the authors observed X-ray reflections consistent with the existence of "troponinbridges", i.e., a combination of cross-bridges bound to the troponin complex. Thus, the similarities between insect flight and vertebrate muscle leave us with the likelihood that passive strain imposed onto the thick-filament of cardiac muscle increases the "activation" of ready-to-weak cross-bridges with direct troponin-bridge formation (and/ or even tropomyosin; Behrmann et al. 2012; actomyosintroponin-tropomyosin bridges). The greater the stretch, the greater the cross-bridge recruitment, and hence a magnified length-dependent response. 


\section{How does increased myosin-binding to actin potentiate greater than normal bound $\mathrm{Ca}^{2+}$ on cTnC?}

Strong-binding cross-bridges appear to promote global conformational changes in the N-lobe of cTnC (Dong et al. 2007). By reconstitution of myofilaments with labeled cTnC, Dong et al. (2007) measured changes on the cTnC structure when myosin binds. Their results support the concept that stronglybound myosin is required to open the structure of the N-lobe of cTnC thereby increasing myofilament $\mathrm{Ca}^{2+}$-sensitivity. This is consistent with earlier observations that show that $\mathrm{Ca}^{2+}$ is released when muscle fibers are shortened and deactivated, implying an active process of cross-bridge detachment and regulation of the $\mathrm{Ca}^{2+}$-affinity of cTnC. Allen and Kurihara (1982) microinjected the $\mathrm{Ca}^{2+}$-luminescent indicator aequorin in isolated papillary and trabeculae muscles, and observed a rise in intracellular $\left[\mathrm{Ca}^{2+}\right]$ following a quick step-release during contraction, which they attributed to dissociation of $\mathrm{Ca}^{2+}$ from the contractile proteins. Housmans et al. (1983) observed similar phenomena. This view was further supported in membrane-permeabilized muscle preparations by Allen and Kentish (1988) who concluded that $\mathrm{Ca}^{2+}$ was actively released from the contractile apparatus. Hofmann and Fuchs (1987b, 1988) also showed that length-dependent changes affect the $\mathrm{Ca}^{2+}$-affinity of $\mathrm{cTnC}$, by reducing the $\mathrm{Ca}^{2+}$-binding affinity of cTnC upon a decrease in sarcomere length. Taken together, these studies indicate that, following fiber lengthening (or shortening), cross-bridge recruitment increases (or decreases) and this potentiates troponin conformational alterations with more (or less) bound $\mathrm{Ca}^{2+}$; arguably, these can be attributed to the mechanosensing role of titin in length-dependent increases (or decreases) in force.

More recently, we have provided evidence that human sarcomeric thin-filament mutations disrupt thin-filament transitions with greater accessibility of myosin-bringing sites on actin, and these are associated with reduced lengthdependence and myofilament $\mathrm{Ca}^{2+}$-sensitization (Sequeira et al. 2013, 2015). It is worth mentioning that a recent study from Dvornikov et al. (2016) puts into question whether thinfilament mutations directly impact myofilament lengthdependent activation. Specifically, they found that the cardiac troponin I (cTnI) R145W mutation (known to cause hypertrophic and restrictive cardiomyopathy) does not (directly) disrupt length-dependent activation. Instead, the authors observed that the cTnI R145W mutation directly impairs cTnIphosphorylation of the two serine sites in humans (serines 23 and 24) responsible for the regulation of length-dependent activation (Dvornikov et al. 2016). The discrepancy in their results is unclear, since, in our studies using human material from a patient with the cTnI R145W mutation (and incubating the cardiomyocytes with Protein Kinase A to exogenously phosphorylate cTnI-S23/S24, validated by specific antibody staining), we did find that the cTnI R145W mutation has the ability by itself to impair length-dependent activation (Sequeira et al. 2013).

\section{Titin-induced strain on Tropomyosin's stiffness increases the Off-On transition of thin-filament units}

The thin-filament functional unit comprises seven actin monomers spanned by one tropomyosin dimer and one cTn complex $\left(\mathrm{A}_{7} \mathrm{TmTn}\right.$ ) (Huxley 1973). $\mathrm{Ca}^{2+}$-binding to $\mathrm{cTnC}$ promotes cTnI detachment from actin and potentiates tropomyosin movement, resulting in the exposure of myosin-binding sites. This movement allows a $\mathrm{Ca}^{2+}$-cooperative activation of the thin-filament with additional recruitment of strongbinding cross-bridges. Structural data suggest that each individual strongly-bound cross-bridge binds to a single regulatory unit $\left(\mathrm{A}_{7} \operatorname{TmTn}\right.$ spanning $\sim 38.5 \mathrm{~nm}$ ), and may regulate tropomyosin movement for up to about three units (covering $\sim 115 \mathrm{~nm}$ ) along the thin-filament in the presence of $\mathrm{Ca}^{2+}$ (Vibert et al. 1997). This has been validated in biochemical studies (Geeves and Lehrer 1994; Maytum et al. 1998).

Tropomyosin increases communication between nearneighboring regulatory units, a property governed by the head-to-tail interaction of tropomyosin (i.e., overlap region) (Hill et al. 1980; Nagashima and Asakura 1982; Heeley et al. 1989; Pan et al. 1989; Geeves and Lehrer 1994). Removal of this overlap reduces cooperative-binding of myosin (Johnson and Smillie 1977; Heeley et al. 1989; Pan et al. 1989). Two recent studies support the idea that $\mathrm{Ca}^{2+}$-cooperative effects are independent of myosin-binding and are strongly associated with the thin-filaments (Sun et al. 2009; Farman et al. 2010). Sun et al. (2009) reconstituted cardiac thin-filaments with fluorescent-labeled $\mathrm{TnC}$ and analyzed changes in the orientation of the structure of troponin. They observed that blebbistatin (which prevents strong-binding formation) had no effect on the Hill coefficient (Sun et al. 2009). Farman et al. (2010) reached similar conclusions, because, in their experiments, blebbistatin decreased $\mathrm{Ca}^{2+}$-sensitivity and force, but did not affect the Hill coefficient. Importantly, the authors reconstituted rat cardiac thin-filaments with a cTnC mutant incapable of binding $\mathrm{Ca}^{2+}$, and observed that both $\mathrm{Ca}^{2+}$-sensitivity and the Hill coefficient were decreased (Farman et al. 2010). They also observed that the effects of the cTnC mutant were greater at shorter $(2.0 \mu \mathrm{m})$ than at longer $(2.2 \mu \mathrm{m})$ sarcomere lengths. The later studies are consistent with recent $\mathrm{X}$-ray structural investigations that also support troponin thin-filament rearrangements upon diastolic lengthening, independent of thick-filament alterations (AitMou et al. 2016). Specifically, Ait-Mou et al. (2016) found that, aside from myosin-strain alterations induced by titin, troponin alterations were also shown to be strain-dependent, and their results support both mechanisms (troponin- and 
myosin-strain dependency) operating simultaneously upon myofilament stretch.

In the study of Farman et al. (2010), the authors attributed thin-filament length-dependent activation due to the ability of tropomyosin to recruit more regulatory units $\left(\mathrm{A}_{7} \mathrm{TmTn}\right)$ upon stretch. Because of the tight crosstalk between troponintropomyosin interactions, the increased stiffness of tropomyosin at longer sarcomere lengths affects three to four $\mathrm{A}_{7} \mathrm{TmTn}$ units in tandem. Conversely, at shorter muscle lengths, tropomyosin is "weakly" stiff, and hence affects fewer thinfilament units (i.e., less troponin structural rearrangements).

When considering how length-dependent alterations influence the cooperative unit size of tropomyosin (presumably via changes in stiffness) by an independent crossbridge component, we suggest that titin may be responsible (Fig. 2). It not only binds to actin at the Z-disc (Trombitás et al. 1997) but also binds both thin-filaments (actin and tropomyosin) in the I-band region (Raynaud et al. 2004). We propose that, upon stretch, titin-binding to tropomyosin at the I-band magnifies the stiffness of tropomyosin's, thereby increasing the functional unit size, independent on its action of cross-bridges (Fig. 2). We recognize that such a mechanism would allow titin to mediate thin-filament activation by affecting the Off-On transition of tropomyosin, troponin structure alterations, and the $\mathrm{Ca}^{2+}$-cooperative size (Fig. 2).

\section{Tryptic digestion of titin}

An interesting result from Fukuda et al. $(2001,2005)$, which shares similarities with the study of Farman et al. (2010), may provide some support to this idea. The authors observed that trypsin digestion of titin does not affect shorter sarcomere lengths $(1.9 \mu \mathrm{m})$ active developed-forces and $\mathrm{Ca}^{2+}$-sensitivity, although stretching to $2.25 \mu \mathrm{m}$ is associated with both force and $\mathrm{Ca}^{2+}$-sensitivity reductions in cardiac muscle preparations (Fukuda et al. 2001, 2005). Based a

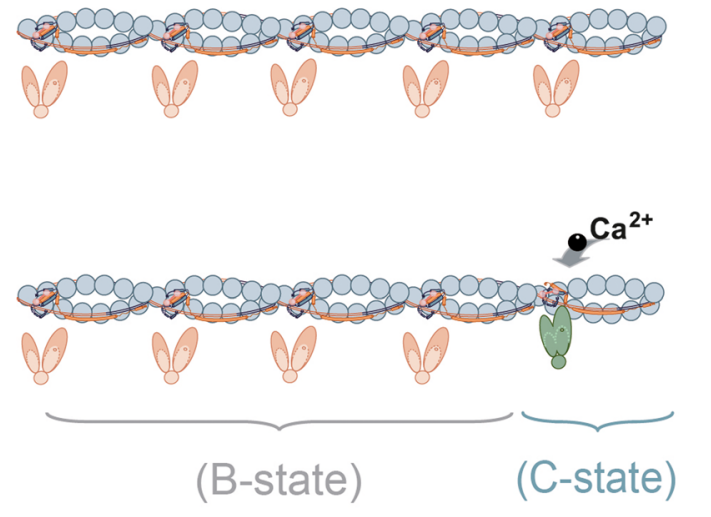

b

b $\quad S L=1.8 \mu \mathrm{m}$

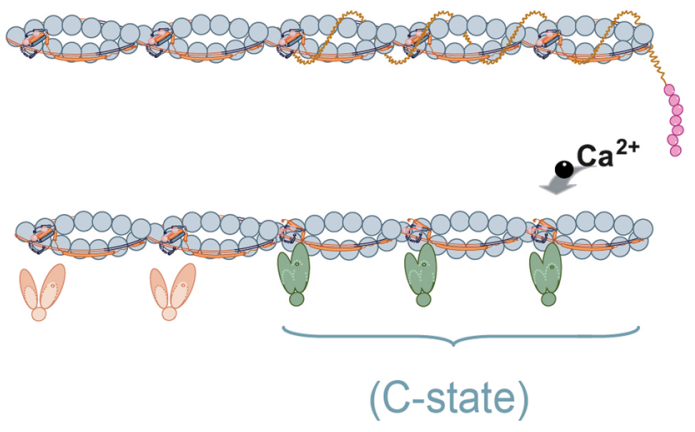

Fig. 2 Proposed scheme for tropomyosin-induced $\mathrm{Ca}^{2+}$-cooperative activation induced by titin. a In the absence of $\mathrm{Ca}^{2+}$ (B-state), tropomyosin sterically blocks the myosin-binding sites on actin. Upon $\mathrm{Ca}^{2+}$ rise, one functional unit comprising of seven actin monomers spanned by one tropomyosin dimer and one cTn complex $\left(\mathrm{A}_{7} \mathrm{TmTn}\right)$ are activated, resulting from $\mathrm{Ca}^{2+}$-binding to $\mathrm{cTnC}$ which leads to tropomyosin movement and the binding of a single weakly-bound cross-bridge (C-state). b, left At shorter sarcomere lengths, tropomyosin-induced stretch by titin increases tropomyosin's stiffness,
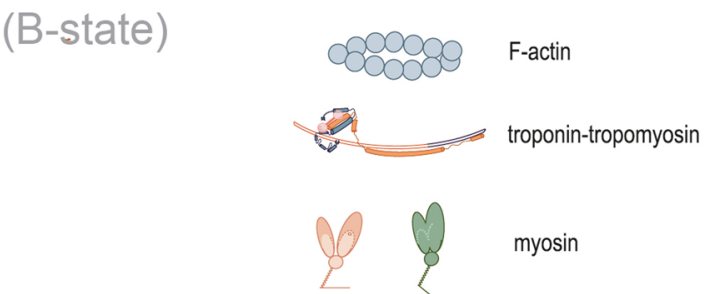

mm- titin

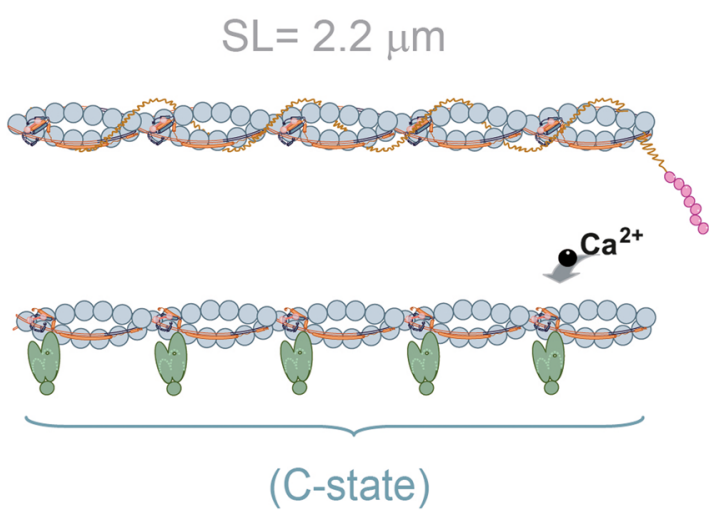

leading to $\mathrm{Ca}^{2+}$-cooperative activation (C-state transition) of up to two additional near-neighboring functional units. b, right At longer sarcomere lengths, an even stiffer tropomyosin induced via greater titin-passive strain at the thin-filaments, additionally activates up to four functional units. Greater amounts of myosin-binding sites are available at longer muscle lengths. This coincides with both the increased transition of ready to weakly-bound cross-bridges imposed by titin at the thick-filament and the approximation of myosin and actin due to reductions of lattice spacing 
on the latter, we speculate that at shorter sarcomere lengths, the normal capacity of tropomyosin to recruit functional units is less pronounced compared to longer sarcomere lengths due to less titin-induced strain on tropomyosin stiffness (i.e., persistence length). We recognize that trypsin digestion of titin at shorter muscle lengths have less effect on $\mathrm{Ca}^{2+}$-activated muscles. Titin-mediated tropomyosin-induced increases in stiffness would generate a long-range activation along the thin-filament (Fig. 2).

In summary the above data support the concept that titin contributes to the Frank-Starling "jigsaw", in an all-atonce manner, following fiber lengthening. Titin appears to mediate length-dependent activation by synergistically affecting tropomyosin-troponin, and its long-range $\mathrm{Ca}^{2+}$ cooperative activation, but also by potentiating cross-bridge's recruitment at the thick-filament with more $\mathrm{Ca}^{2+}$ bound on $\mathrm{cTnC}$.

\section{Discussion}

Over a century of research on the Frank-Starling Law, at the whole heart and the cardiomyocyte level, has vastly advanced our understanding of the fundamental basis of muscle. In particular, the elusive relationship between muscle lengthening and the sensitivity to $\mathrm{Ca}^{2+}$ ions appears to primarily involve the giant protein titin. It can regulate a composite of several synergistic processes that result in the formation of stronglybound cross-bridges in thick-filaments that enhance $\mathrm{Ca}^{2+}$-affinity on cTnC. Not limited to, and open for debate, titin binding in the I-band region may increase the $\mathrm{Ca}^{2+}$-cooperative unit size by regulating the location (and persistence length) of tropomyosin and $\mathrm{Ca}^{2+}$-activation. Overall, titin mediation of length-dependent activation appears to: (1) orchestrate alterations in interfilament lattice spacing that approximate myosin and actin, and (2) induce strain in the thick-filaments that promotes the formation of strongbinding cross-bridges and cTnC alterations; while finally (3) titin-induced strain in the thin-filament increases the $\mathrm{Ca}^{2+}$-cooperative unit size.

Acknowledgments We acknowledge support from the Amsterdam Cardiovascular Sciences and the Netherlands organization for scientific research (NWO; VIDI grant 91711344).

\section{Compliance with ethical standards}

Conflict of interest Vasco Sequeira declares that he has no conflicts of interest. Jolanda van der Velden declares that she has no conflicts of interest.

Ethical approval This article does not contain any studies with human participants or animals performed by any of the authors.
Open Access This article is distributed under the terms of the Creative Commons Attribution 4.0 International License (http:// creativecommons.org/licenses/by/4.0/), which permits unrestricted use, distribution, and reproduction in any medium, provided you give appropriate credit to the original author(s) and the source, provide a link to the Creative Commons license, and indicate if changes were made.

\section{References}

Ait-Mou Y, Hsu K, Farman GP, Kumar M, Greaser ML, Irving TC, de Tombe PP (2016) Titin strain contributes to the Frank-Starling law of the heart by structural rearrangements of both thin- and thickfilament proteins. Proc Natl Acad Sci U S A 113:2306-2311

Allen DG, Kentish JC (1985) The cellular basis of the length-tension relation in cardiac muscle. J Mol Cell Cardiol 17:821-840

Allen D, Kentish J (1988) Calcium concentration in the myoplasm of skinned ferret ventricular muscle following changes in muscle length. J Physiol 407:489-503

Allen DG, Kurihara S (1982) The effects of muscle length on intracellular calcium transients in mammalian cardiac muscle. J Physiol 327:79-94

Behrmann E, Müller M, Penczek Pawel A, Mannherz Hans G, Manstein Dietmar J, Raunser S (2012) Structure of the rigor actintropomyosin-myosin complex. Cell 150:327-338

Cazorla O, Vassort G, Garnier D, Le Guennec J-Y (1999) Length modulation of active force in tat cardiac myocytes: is titin the sensor? J Mol Cell Cardiol 31:1215-1227

Cazorla O, Wu Y, Irving TC, Granzier H (2001) Titin-based modulation of calcium sensitivity of active tension in mouse skinned cardiac myocytes. Circ Res 88:1028-1035

Dong W-J, Jayasundar JJ, An J, Xing J, Cheung HC (2007) Effects of PKA phosphorylation of cardiac troponin I and strong crossbridge on conformational transitions of the $\mathrm{N}$-domain of cardiac troponin $\mathrm{C}$ in regulated thin filaments. Biochemistry 46:9752-9761

Dvornikov AV, Smolin N, Zhang M, Martin JL, Robia SL, de Tombe PP (2016) Restrictive cardiomyopathy troponin I R145W mutation does not perturb myofilament length-dependent activation in human cardiac sarcomeres. J Biol Chem 291:21817-21828

Farman GP, Allen EJ, Schoenfelt KQ, Backx PH, de Tombe PP (2010) The role of thin filament cooperativity in cardiac length-dependent calcium activation. Biophys J 99:2978-2986

Farman GP, Gore D, Allen E, Schoenfelt K, Irving TC, de Tombe PP (2011) Myosin head orientation: a structural determinant for the Frank-Starling relationship. Am J Phys 300:2155-2160

Freiburg A, Gautel M (1996) A molecular map of the interactions between titin and myosin-binding protein C. Implications for sarcomeric assembly in familial hypertrophic cardiomyopathy. Eur J Biochem 235:317-323

Fuchs F, Wang Y-P (1996) Sarcomere length versus interfilament spacing as determinants of cardiac myofilament $\mathrm{Ca}^{2+}$ sensitivity and $\mathrm{Ca}^{2+}$ binding. J Mol Cell Cardiol 28:1375-1383

Fukuda N, Kajiwara H, Si I, Kurihara S (2000) Effects of MgADP on length dependence of tension generation in skinned rat cardiac muscle. Circ Res 86:e1-e6

Fukuda N, Sasaki D, Si I, Kurihara S (2001) Length dependence of tension generation in rat skinned cardiac muscle: role of titin in the Frank-Starling mechanism of the heart. Circulation 104:1639-1645

Fukuda N, Wu Y, Farman G, Irving TC, Granzier H (2003) Titin isoform variance and length dependence of activation in skinned bovine cardiac muscle. J Physiol 553:147-154 
Fukuda N, Wu Y, Farman G, Irving T, Granzier H (2005) Titin-based modulation of active tension and interfilament lattice spacing in skinned rat cardiac muscle. Pflugers Arch 449:449-457

Fürst DO, Osborn M, Nave R, Weber K (1988) The organization of titin filaments in the half-sarcomere revealed by monoclonal antibodies in immunoelectron microscopy: a map of ten nonrepetitive epitopes starting at the $\mathrm{Z}$ line extends close to the M line. J Cell Biol 106: $1563-1572$

Geeves MA, Lehrer SS (1994) Dynamics of the muscle thin filament regulatory switch: the size of the cooperative unit. Biophys J 67 : 273-282

Godt R, Maughan D (1977) Swelling of skinned muscle fibers of the frog. Biophys J 19:103-116

Godt RE, Maughan DW (1981) Influence of osmotic compression on calcium activation and tension in skinned muscle fibers of the rabbit. Pflugers Arch 391:334-337

Hanft LM, Biesiadecki BJ, McDonald KS (2013) Length dependence of striated muscle force generation is controlled by phosphorylation of cTnI at serines 23/24. J Physiol 591:4535-4547

Head JG, Ritchie MD, Geeves MA (1995) Characterization of the equilibrium between blocked and closed states of muscle thin filaments. Eur J Biochem 227:694-699

Heeley DH, Smillie LB, Lohmeier-Vogel EM (1989) Effects of deletion of tropomyosin overlap on regulated actomyosin subfragment 1 ATPase. Biochem J 258:831-836

Hibberd M, Jewell B (1982) Calcium- and length-dependent force production in rat ventricular muscle. J Physiol 329:527-540

Hill TL, Eisenberg E, Greene L (1980) Theoretical model for the cooperative equilibrium binding of myosin subfragment 1 to the actintroponin-tropomyosin complex. Proc Natl Acad Sci U S A 77:31863190

Hofmann PA, Fuchs F (1987a) Effect of length and cross-bridge attachment on $\mathrm{Ca} 2+$ binding to cardiac troponin C. Am J Phys 253:C90 C96

Hofmann PA, Fuchs F (1987b) Evidence for a force-dependent component of calcium binding to cardiac troponin C. Am J Phys 253: C541-C546

Hofmann PA, Fuchs F (1988) Bound calcium and force development in skinned cardiac muscle bundles: effect of sarcomere length. J Mol Cell Cardiol 20:667-677

Housmans PR, Lee NK, Blinks (1983) Active shortening retards the decline of the intracellular calcium transient in mammalian heart muscle. Science 221:159-161

Huxley HE (1973) Muscle 1972: progress and problems. Cold Spring Harb Symp Quant Biol 37:689-693

Irving TC, Konhilas J, Perry D, Fischetti R, de Tombe PP (2000) Myofilament lattice spacing as a function of sarcomere length in isolated rat myocardium. Am J Phys 279:H2568-H2573

Johnson P, Smillie LB (1977) Polymerizability of rabbit skeletal tropomyosin: effects of enzymic and chemical modifications. Biochemist 16:2264-2269

Kampourakis T, Sun Y-B, Irving M (2016) Myosin light chain phosphorylation enhances contraction of heart muscle via structural changes in both thick and thin filaments. Proc Natl Acad Sci U S A 113: E3039-E3047

Konhilas JP, Irving TC, de Tombe PP (2002a) Length-dependent activation in three striated muscle types of the rat. J Physiol 544:225-236

Konhilas JP, Irving TC, de Tombe PP (2002b) Myofilament calcium sensitivity in skinned rat cardiac trabeculae: role of interfilament spacing. Circ Res 90:59-65

Konhilas JP, Irving TC, Wolska BM, Jweied EE, Martin AF, Solaro RJ, de Tombe PP (2003) Troponin I in the murine myocardium: influence on length-dependent activation and interfilament spacing. J Physiol 547:951-961

Labeit S, Kolmerer B (1995) Titins: giant proteins in charge of muscle ultrastructure and elasticity. Science 270:293-296
Linari M, Brunello E, Reconditi M, Fusi L, Caremani M, Narayanan T, Piazzesi G, Lombardi V, Irving M (2015) Force generation by skeletal muscle is controlled by mechanosensing in myosin filaments. Nature 528:276-279

Malinchik S, Xu S, Yu LC (1997) Temperature-induced structural changes in the myosin thick filament of skinned rabbit psoas muscle. Biophys J 73:2304-2312

Martyn DA, Gordon AM (1988) Length and myofilament spacingdependent changes in calcium sensitivity of skeletal fibres: effects of $\mathrm{pH}$ and ionic strength. J Muscle Res Cell Motil 9:428-445

Maruyama K, Yoshioka T, Higuchi H, Ohashi K, Kimura S, Natori R (1985) Connectin filaments link thick filaments and $\mathrm{Z}$ lines in frog skeletal muscle as revealed by immunoelectron microscopy. J Cell Biol 101:2167-2172

Mateja RD, Greaser ML, de Tombe PP (2012) Impact of titin isoform on length dependent activation and cross-bridge cycling kinetics in rat skeletal muscle. Biochim Biophys Acta 1833:804-811

Maytum R, Lehrer SS, Geeves MA (1998) Cooperativity and switching within the three-state model of muscle regulation. Biochemistry 38 : $1102-1110$

McDonald KS, Moss RL (1995) Osmotic compression of single cardiac myocytes eliminates the reduction in $\mathrm{Ca} 2+$ sensitivity of tension at short sarcomere length. Circ Res 77:199-205

Moss L, Swinford A, Greaser M (1983) Alterations in the $\mathrm{Ca}^{2+}$ sensitivity of tension development by single skeletal muscle fibers at stretched lengths. Biophys J 43:115-119

Nagashima H, Asakura S (1982) Studies on co-operative properties of tropomyosin-actin and tropomyosin-troponin-actin complexes by the use of N-ethylmaleimide-treated and untreated species of myosin subfragment 1. J Mol Biol 155:409-428

Pan BS, Gordon AM, Luo ZX (1989) Removal of tropomyosin overlap modifies cooperative binding of myosin S-1 to reconstituted thin filaments of rabbit striated muscle. J Biol Chem 264:8495-8498

Perz-Edwards RJ, Irving TC, Baumann BAJ, Gore D, Hutchinson DC, Kržič U, Porter RL, Ward AB, Reedy MK (2011) X-ray diffraction evidence for myosin-troponin connections and tropomyosin movement during stretch activation of insect flight muscle. Proc Natl Acad Sci U S A 108:120-125

Raynaud F, Astier C, Benyamin Y (2004) Evidence for a direct but sequential binding of titin to tropomyosin and actin filaments. Biochim Biophys Acta 1700:171-178

Reconditi M, Caremani M, Pinzauti F, Powers JD, Narayanan T, Stienen GJM, Linari M, Lombardi V, Piazzesi G (2017) Myosin filament activation in the heart is tuned to the mechanical task. Proc Natl Acad Sci U S A 114:3240-3245

Sequeira V, van der Velden J (2015) Historical perspective on heart function: the Frank-Starling Law. Biophys Rev 7:421-447

Sequeira V, Wijnker PJM, Nijenkamp LLAM, Kuster DWD, Najafi A, Witjas-Paalberends ER, Regan JA, Boontje N, ten Cate FJ, Germans T, Carrier L, Sadayappan S, van Slegtenhorst MA, Zaremba R, Foster DB, Murphy AM, Poggesi C, dos Remedios C, Stienen GJM, Ho CY, Michels M, van der Velden J (2013) Perturbed length-sependent activation in human hypertrophic cardiomyopathy with missense sarcomeric gene mutations. Circ Res 112:1491-1505

Sequeira V, Najafi A, Wijnker P, Dos Remedios C, Michels M, Kuster DWD, van der Velden J (2015) ADP-stimulated contraction: a predictor of thin-filament activation in cardiac disease. Proc Natl Acad Sci U S A 112:E7003-E7012

Smith SH, Fuchs F (1999) Effect of ionic strength on length-dependent $\mathrm{Ca}^{2+}$ activation in skinned cardiac muscle. J Mol Cell Cardiol 31: 2115-2125

Sun Y-B, Lou F, Irving M (2009) Calcium- and myosin-dependent changes in troponin structure during activation of heart muscle. J Physiol 587:155-163

Terui T, Sodnomtseren M, Matsuba D, Udaka J, Si I, Ohtsuki I, Kurihara S, Fukuda N (2008) Troponin and titin coordinately regulate length- 
dependent activation in skinned porcine ventricular muscle. J Gen Physiol 131:275-283

Trombitás K, Greaser ML, Pollack GH (1997) Interaction between titin and thin filaments in intact cardiac muscle. J Muscle Res Cell Motil 18:345-351

Vibert P, Craig R, Lehman W (1997) Steric-model for activation of muscle thin filaments. J Mol Biol 266:8-14

Wakabayashi K, Sugimoto Y, Tanaka H, Ueno Y, Takezawa Y, Amemiya Y (1994) X-ray diffraction evidence for the extensibility of actin and myosin filaments during muscle contraction. Biophys J 67:2422-2435

Wang Y-P, Fuchs F (1995) Osmotic compression of skinned cardiac and skeletal muscle bundles: effects on force generation, $\mathrm{Ca}^{2+}$ sensitivity and $\mathrm{Ca}^{2+}$ binding. J Mol Cell Cardiol 27:1235-1244
Wijnker PJM, Sequeira V, Foster DB, Li Y, dos Remedios CG, Murphy AM, Stienen GJM, van der Velden J (2014) Length-dependent activation is modulated by cardiac troponin I bisphosphorylation at Ser23 and Ser24 but not by Thr143 phosphorylation. Am J Phys 306:H1171-H1181

Xu S, Kress M, Huxley HE (1987) X-ray diffraction studies of the structural state of crossbridges in skinned frog sartorius muscle at low ionic strength. J Muscle Res Cell Motil 8:39-54

Xu S, Malinchik S, Gilroy D, Kraft T, Brenner B, Yu LC (1997) X-ray diffraction studies of cross-bridges weakly bound to actin in relaxed skinned fibers of rabbit psoas muscle. Biophys J 73:22922303

Zoghbi ME, Woodhead JL, Moss RL, Craig RW (2008) Threedimensional structure of vertebrate cardiac muscle myosin filaments. Proc Natl Acad Sci U S A 105:2386-2390 